\title{
Corela
}

Cognition, représentation, langage

14-1 | 2016

Vol. $14, n^{\circ} 1$

\section{L'adjectif appréciatif nice : observations sur la nature variable de la prédication}

\section{Christopher Desurmont}

\section{OpenEdition}

\section{Journals}

Édition électronique

URL : http://journals.openedition.org/corela/4566

DOI : $10.4000 /$ corela.4566

ISSN : 1638-573X

Éditeur

Cercle linguistique du Centre et de l'Ouest - CerLICO

Référence électronique

Christopher Desurmont, «L'adjectif appréciatif nice : observations sur la nature variable de la prédication », Corela [En ligne], 14-1 | 2016, mis en ligne le 16 juin 2016, consulté le 19 avril 2019. URL http://journals.openedition.org/corela/4566 ; DOI : 10.4000/corela.4566

Ce document a été généré automatiquement le 19 avril 2019

\section{(c) (1) (2)}

Corela - cognition, représentation, langage est mis à disposition selon les termes de la licence Creative Commons Attribution - Pas d'Utilisation Commerciale - Partage dans les Mêmes Conditions 4.0 International. 


\title{
L'adjectif appréciatif nice : observations sur la nature variable de la prédication
}

\author{
Christopher Desurmont
}

\section{Introduction}

1 Cet article porte sur les divers emplois et interprétations de l'adjectif nice dans une analyse uniquement synchronique. Les données diachroniques n'entrent pas en considération dans cette analyse. Cependant, signalons ceci. Nice vient du moyen anglais, qui lui vient du vieux français nice ('simple, foolish, ignorant'), lui-même du latin nescius ('ignorant, not knowing') : ne ('not') + scire (' to know'). Au fil du temps, la signification a pu varier considérablement, et cet adjectif en vient à recevoir une connotation positive, notamment avec le sens /agreable/ ou /delightful/ en tout cas à partir du $18^{\text {ème }}$. La polysémie de nice va augmenter au point de permettre à cet adjectif appréciatif de s'appliquer pratiquement à tout objet référentiel jugé positif (par le locuteur ou par la communauté dans son ensemble), selon les critères suivants (liste non exhaustive) : de / beauté/ (a nice sight/view/dress), d'/agrément/ ( nice idea), de la /morale/ ou de l'/affectivité/ (a nice decision/attitude/person). L'adjectif nice est donc d'un emploi très fréquent, occupant une place à part dans la classe des adjectifs appréciatifs ; sa signification en discours - à connotation posive -, variant en fonction du contexte : selon la référence de la tête nominale $\mathrm{N}$ s'il est le seul adjectif épithète ; selon le contenu référentiel des autres adjectifs épithètes s'il y en a; et si nice est attribut du sujet, selon la nature et la référence du SN-sujet cible de la prédication.

2 L'analyse synchronique est ici basée principalement sur deux couples de concepts: prédicat d'individu/prédicat épisodique (individual/stage level predicates: ILP/SLP), interprétation intersective/subsective (non intersective) du prédicat. À titre de comparaison, et seulement lorsque l'analyse de l'adjectif nice le demande, ces critères 
sont également sollicités brièvement dans la description d'autres adjectifs (eager, smart, good, beautiful). L'un des buts ici est d'essayer de montrer l'utilité de ces outils conceptuels dans l'étude des adjectifs "appréciatifs", et cela à travers celle d'un exemplaire particulier.

Un adjectif A est intersectif s'il réussit le test suivant: si «Cet X est un AN ou un NA » implique : 1 . cet $\mathrm{X}$ est un $\mathrm{N}$, et 2 . Cet $\mathrm{X}$ est $\mathrm{A}$. «Cet objet est une voiture rouge », implique 1. Cet objet est une voiture, 2. Cet objet est rouge. L'objet dénoté par $\mathrm{X}$ appartient à la fois à la classe des objets entrant dans l'extension de $\mathrm{N}$ et dans celui de $\mathrm{A}$. Les adjectifs subsectifs (non intersectifs) - de taille et de dimension par exemple - ne réussissent pas ce test de l'intersectivité. Ainsi, «Cette chose est une petite fleur » implique 1. Cette chose est une fleur, mais n'implique pas 2. \# Cette chose est petite, car une fleur est petite (ou grande) POUR une fleur.

4 La partie (1) s'appuie sur trois tests classiques permettant de différencier les prédicats épisodiques (SLP) des prédicats d'individu (ILP), et fait valoir que les SA-prédicatifs [nice] et $\left[_{S A}\right.$ nice $\left[{ }_{S P}\right.$ with $\left.\left.S N\right]\right]$ seraient tous deux de type SLP, alors que le SA $\left[_{S A}\right.$ nice $\left[{ }_{S P}\right.$ of $\left.\left.S N\right]\right]$ serait de type ILP, tout comme le SN-prédicatif [a nice N]. La partie (2) est consacrée à nice en fonction épithète (attributive), et la partie (3) à nice en fonction attribut (predicative). La partie (4) fait valoir une paradoxale compatibilité entre le SN-prédicatif (ILP) [a nice N] et un $\mathrm{SN}$-sujet dont la tête nominale est complétée par une relative à contenu événementiel. La partie (5), basée sur les concepts d'intersectivité/subsectivité, montre que dans le SNprédicatif [a nice $N$ ], nice reçoit parfois une lecture intersective, mais le plus souvent une lecture subsective, et que les deux lectures peuvent parfois cohabiter. La partie (6) examine la fonction synthétique de nice comme épithète, soit seule devant la tête nominale $\mathrm{N}$, soit suivie d'un ou de plusieurs adjectifs descriptifs.

1. Prédicat d'individu / prédicat épisodique ${ }^{1}$

(individual/stage level predicate: ILP/SLP)

5 Si un énonciateur dit She's a nice person, il/elle effectue ce qui semble être une prédication d'individu (individual level predication: ILP), basée sur la validation antérieure de procès significatifs, interprétés comme autant d'indices de la qualité /nice/ : procès ayant fait l'objet d'appréciations reposant sur des systèmes de valeurs ou de préférences.

Une prédication d'individu (ILP) signifie une propriété non bornée de l'objet (We're human beings; you're intelligent; she's knowledgeable). Une prédication épisodique (SLP) signifie une propriété contingente, limitée dans le temps : They're away (for the holidays), He's in (right now). La plupart des adjectif appréciatifs ${ }^{2}$ peuvent être utilisés des deux manières.

Divers tests ont été proposés pour distinguer les prédicats SLP des prédicats ILP. En voici trois ; cf. Carlson (1980), et Kertz (2006) :

8 TEST 1. Les prédicats SLP sont compatibles avec un adverbe de fréquence, les prédicats ILP ne le sont pas :

1. *Copland was often smart to write about his compositions. A. appréciatif

2. Copland was often eager to write about his compositions. A. d'attitude (SLP) ${ }^{4}$

Comparons à présent :

3. She is often $\left[_{S A}\right.$ nice $]$

4. She was often $\left[{ }_{S A}\right.$ nice $]$.

5. ?She was $\left[_{\mathrm{SN}}\right.$ a nice person] that day.

6. ?She is often [ ${ }_{S N}$ a nice person].

7. ? ?She was often $\left[_{S N}\right.$ a nice person] that day. 
$\left.111^{\circ}\right)$ que le SA-prédicatif [nice] est compatible avec l'adverbe often quel que soit le temps verbal ; ce qui implique une prédication de type SLP en (3) et (4); que le $\mathrm{SN}$ [a nice N] ne peut être prédicatif s'il y a adjonction au SV d'un adverbe de fréquence; ce qui implique que le SN-prédicatif n'a pas vocation à participer à une prédication de type SLP, et qu'il serait donc de type ILP.

ces observations, on peut essayer d'expliquer la différence d'acceptabilité entre (7) et (5). Celle-ci tient manifestement à la présence ou l'absence de l'adverbe de fréquence often. Sa présence en (7) signifie que la prédication concerne un nombre pluriel d'actions et d'instants dans le cadre de «that day », mais si le SN [a nice $N$ ] est bien de type ILP, il n'est pas qualifié pour jouer ce rôle prédicatif. Pour les locuteurs qui accepteraient davantage l'énoncé (5), l'explication pourrait être la suivante. En l'absence de l'adverbe de fréquence often, la prédication traduirait un jugement sur la personne elle-même plutôt que sur ses diverses actions ce jour-là, même si celles-ci ont servi d'indices de la qualité /nice/, et même si la prédication est associée à un circonstant qui en limite la portée temporelle; ce qui est néanmoins paradoxal pour une prédication ILP, d'où l'incertitude sur le degré d'acceptabilité de cet énoncé. signification que (13) avec interprétation de type SLP; et elle est en tout cas moins inacceptable que (8).

21 Conclusions :

22 (d) (10) et (11) montrent que ni le SA [nice] ni le SN [a nice N] ne peuvent être directement enchâssés sous un verbe de perception; ce qui suggère qu'ils ne relèvent ni l'un ni l'autre de la prédication SLP ; ce qui contredit la conclusion (a), mais conforte la conclusion (b). Cependant, (10) est corrigé en insérant le verbe be au participe présent: We saw her being nice (i.e. acting nicely), avec un prédicat manifestement de type SLP.

(e) (12) montre que nice peut être enchâssé sous un verbe de perception s'il est postmodifié par le SP [with SN]; cette observation permet d'émettre l'hypothèse - en faveur de la conclusion (a) - que l'énoncé (3) peut être lu comme une structure elliptique : she is often $\left[_{S A}\right.$ nice $]=$ she is often $\left[_{S A}\right.$ nice $\left[_{S P}[\varnothing]\right]$; i.e., she is often $\left[_{S A}\right.$ nice $\left[_{S P}\right.$ with 
everyone/you/us]]. L'intérêt supplémentaire de cette hypothèse est qu'elle aménage une position syntaxique vide, étiquetée SP, qui permet toute latitude interprétative, selon les contextes.

TEST 3. Les prédicats SLP et ILP sont tous deux compatibles avec une localisation spatiale ou temporelle, mais seuls les prédicats SLP sont compatibles avec l'expression circonstancielle d'une dimension spatio-temporelle (a spatio-temporal expanse or dimension ) :

14. Yesterday at the meeting, John was smart to volunteer. ILP

15. Yesterday at the meeting, John was eager to help. SLP

16.* All the way from London to Brighton, John was smart to drive. *ILP

17. All the way from London to Brighton, John was eager to drive. SLP

Comparons :

18. Yesterday at the meeting, she was nice.

19. ? ? Yesterday at the meeting, she was a nice person.

20. All the way from home to the station, she was nice. SLP

? ?All the way from home to the station, she was a nice person. *ILP

Conclusions:

(g) Le SA-prédicatif [nice] (attribut du sujet) semble bien être un prédicat de type SLP.

(h) Le SN-prédicatif [a nice N] semble être un prédicat de type ILP.

Revenons un instant sur la nature de la prédication du SA-prédicatif [nice] en observant une différence avec les adjectifs appréciatifs comme smart. Dans les structures personnelles, l'un peut être suivi d'une proposition infinitive (a), mais pas l'autre (c) :

a) He was $\left[{ }_{S A}\right.$ smart $]$ to have negotiated with them. ILP

b) It was $\left[{ }_{\mathrm{SA}}\right.$ smart [of him]] to have negotiated with them. ILP

c) *He was $\left[_{\mathrm{SA}}\right.$ nice $]$ to have come. *SLP

d) It was $[\mathrm{SA}$ nice [of him]] to have come. ILP

Selon l'analyse de Kertz (2006), (a) serait une prédication de type ILP, et la proposition infinitive serait un constituant adjoint au nœud SA (et donc ni une adjonction au nœud SV, ni le complément de A). Selon le point de vue de Yagi (2006), adopté ici pour l'analyse de (b) et (d) ci-dessus, sa phrase It is silly of him to believe her (10b) relèverait de la prédication de type ILP: "The preposition of as in (10b) is used to specify who is silly, in other words, (10b) is a statement about him, not the event as a whole". L'agrammaticalité de (c) peut alors être expliquée sur la base de deux postulats :

$\left.1^{\circ}\right)$ La proposition infinitive [PRO to have come] serait une adjonction au nœud SA ;

$2^{\circ}$ ) Le SA-prédicatif [nice] serait un prédicat SLP (comme nous l'avons soutenu plus haut).

L'agrammaticalité de (c) s'expliquerait alors en faisant valoir que l'adjectif appréciatif en (c) devrait être de type ILP - comme l'adjectif smart en (a) - , alors que le SA-prédicatif [nice] est de type SLP.

\section{Nice comme épithète prénominale (attributive)}

4 Selon les critères de Greenbaum et Quirk (1990), nice appartiendrait à la classe (hétérogène) des "adjectifs centraux» (central adjectives), car pouvant être aussi bien épithète prénominale qu'attribut du sujet (attributive + predicative): 'The ability to function attributively and the ability to function predicatively are central features of adjectives.' (p. 129). 

propriété de l'objet, et qui se comprend comme l'expression d'un jugement personnel, ce qui fait dire qu'il s'agirait d'un "adjectif subjectif » (i.e. speaker-oriented); mais cette observation doit être nuancée. Si nice est épithète prénominale (attributive), il hérite d'une " propriété positionnelle $»^{6}$ bien connue (cf. Bolinger, 1967) : A confère au référent de AN le statut de sous-ensemble de la classe d'objets dénotés par N. Cela se comprend aisément pour l'adjectif descriptif intersectif (red car), (wooden bridge), ou pour l'adjectif dénominal relationnel/associatif (southern view). Cela commence en revanche à poser problème pour certains emplois des adjectifs descriptifs relatifs ou subsectifs (long trip) ; mais plus encore pour les adjectifs appréciatifs comme nice dont l'imprécision, la « relativité » ou la «subsectivité » n'est pas une question de mesure quantitative. Comment considérer la propriété /nice/ comme signe distinctif (d'un sous-ensemble) si l'adjectif appréciatif est un " adjectif non-spécifique » dans le sens donné à ce terme (par exemple) par I. Thomas : «(...) les adjectifs non-spécifiques (ANS), c'est-à-dire ceux qui peuvent décrire les propriétés de n'importe quel objet, indépendamment de leur statut catégoriel (...) $»^{7}$

des voitures rouges, à celle des voitures, et à celle des objets rouges : l'adjectif de couleur est donc intersectif. S'agissant des adjectifs descriptifs subsectifs (taille, dimension ...), comme dans le SN [a small house], le référent n'appartient pas à la classe des «petites entités " car cette classe n'existe pas, la notion de dimension étant relative; mais la subsectivité de l'adjectif descriptif de dimension (small) n'empêche pas le référent du SN [a small house] d'appartenir à la «classe des petites maisons » avec le trait distinctif de / petitesse/ (fût-il relatif/subsectif).

Les dictionnaires décrivent la notion /nice/ au moyen de synonymes, et en multipliant les exemples avec le SN [a nice N]. Par exemple, l'entrée du dictionnaire Cambridge Advanced Dictionary, Third Edition, 2008, commence ainsi :

NICE adjective PLEASANT 1. E pleasant, enjoyable or satisfactory: Did you have a nice holiday? Hace a nice day/time ! (où la lettre majuscule E signifie : Essential : the most common and useful words in English.)

Le dénominateur commun à l'ensemble des synonymes est d'exprimer une propriété positive ; plus exactement, une propriété agissant sur le sujet (observateur ou expérient) d'une façon ressentie et jugée comme positive. Compte tenu de la "plasticité » de l'adjectif nice dont la signification varie avec chaque contexte, on peut se demander si AN continue ici de désigner un sous-ensemble préconstruit. La réponse semble être oui, et cela précisément en vertu d'une « propriété positionnelle » qui signifie que l'existence du type d'objet dénoté par AN en anglais (où A est « prénominal ») est préconstruite, et que par conséquent l'énonciateur ne fait que relayer une image/représentation. À l'appui de ceci, le fait que dans la plupart des contextes, l'épithète nice en position prénominale peut «faire sens » à elle seule (a nice cake/day/time/trip/walk, etc.), même si dans d'autres exemples, la signification voulue est fixée par la présence filtrante d'une série d'autres qualifications :

22. heating and water heating will turn itself on. So you come home to a nice warm, brightly lit home with plenty of piping hot water to soak-away the dust.

23. a nice warm, brightly lit home [with plenty of piping hot water [to soak-away the dust]]. 
l'extension est plus faible que celle de la classe des [girls]. Dès lors que l'unité d'une classe est fragmentée par la désignation d'un ensemble singularisé par une propriété distinctive (ici : /nice/), se forme automatiquement l'image d'un autre sous-ensemble n'ayant pas cette propriété. Ainsi, la classe des [girls] est implicitement représentée en (24) comme étant formée d'au moins deux ensembles, E1 et E2 ; l'un ayant le trait distinctif /nice/ (E1), l'autre ne l'ayant pas (E2). Se pose alors la question: s'il existe un sous-ensemble dans la classe des filles qui ne possède pas le trait /nice/, cela signifie-t-il qu'il possède un trait négatif antonyme de nice, tel que /nasty/ ? Les adjectifs « évaluatifs » impliquent-ils nécessairement une conception strictement dichotomique du jugement appréciatif? Comment interpréter la négation dans la propriété /not nice/? Une représentation strictement dichotomique donnerait le résultat suivant:

\begin{tabular}{|l|l|l|}
\hline 25. & propriété p & non-p \\
\hline & brave & not brave ( = cowardly) \\
\hline & clever & not clever ( = stupid) \\
\hline & good & not good ( = evil) \\
\hline & nice & not nice ( = nasty) \\
\hline & polite & not polite ( = rude ) \\
\hline & stupid & not stupid ( = intelligent ) \\
\hline & wise & not wise ( = foolish) \\
\hline
\end{tabular}

Cependant, la grande majorité des adjectifs appréciatifs sont gradables (fairly nice, quite nice, very nice), ce qui implique que les objets qualifiés par un adjectif appréciatif, et ici par la propriété /nice/, peuvent l'être de plus de deux manières.

La scalarité de la notion /nice/ se conçoit autour d'une valeur type, et selon le contexte. Ainsi, le SN [nice weather] dénote un certain ensoleillement; [a nice bed] un lit suffisamment confortable; [a nice idea] une idée assez généreuse ou utile (selon le contexte) pour être prise en considération, et toute modalisation de nice induit l'image d'un exemplaire entretenant avec l'occurrence-type du couple AN un certain degré d'altérité qualitative, même si l'objet est encore représenté comme ayant la propriété p. L'existence même d'expressions comme quite nice weather, quite a nice bed, a fairly nice person, (expressions nuancées par modalisation adverbiale) montre à l'évidence que le langage fournit les outils nécessaires à une représentation non strictement dichotomique des domaines notionnels en question. 
Comparons à présent :

26. She's a nice person.

27. She's a very nice person.

L'épithète dans le SN-prédicatif [a nice $N$ ] serait un prédicat de type ILP (tout comme le $\mathrm{SN}$-prédicatif lui-même), car le jugement porte sur la personne, et non pas directement sur les actes ou les comportements qui ont pu motiver l'expression de ce jugement. Ainsi, l'adjectif appréciatif (ILP) nice en (26) se présente comme une propriété définitive.

L'énoncé (26) induit une vision dichotomique divisant la classe des personnes en deux ensembles (nice/not nice). Cependant, dans certains contextes, la qualité inverse (not nice) est presque entièrement occultée, comme par exemple dans l'énoncé suivant: I wouldn't mind a nice cup of tea. En effet, ici le locuteur ne cherche aucunement à signifier que ce qu'il/elle veut est une bonne plutôt qu'une mauvaise tasse de thé, même si cette opposition peut exister dans la réalité. L'énoncé signifie plutôt ceci : I wouldn't mind (i.e. I'd really like) a cup of tea right now, knowing that it will most probably be a nice one and that I'll enjoy it. L'adjectif nice ici n'a donc pas la même valeur contrastive qu'en (26).

La prédication adjectivale en (26) et (27) serait de type ILP (prédication d'individu). Cependant, en (27), la personne est définie au moyen d'un prédicat (nice) que l'énonciateur a cru devoir modaliser (very nice), n'ayant pas voulu ou pas pu se contenter de la notion /nice/ inaltérée, communiquant ainsi le message suivant : «la propriété / nice/ ne peut être prédiquée telle quelle pour la personne en question ». La modalisation par l'adverbe d'intensité very en (27) - qui montre que nice est un adjectif gradable - ne permet pas forcément d'améliorer l'image de la /gentillesse/ sur l'échelle scalaire ; autrement dit, a very nice person n'est pas forcément plus gentille que $a$ nice person. Cette observation implique la nécessité (généralement reconnue) de ne pas confondre les concepts de /scalarité/ et de /gradabilité/. Ajoutons ici que la prédication adjectivale en (27), contrairement à celle en (26), peut en contexte approprié être utilisée de façon contrefactuelle si l'adverbe de degré very est accentué et mis au service de l'ironie.

\section{Nice comme attribut du sujet (predicative)}

51 Se pose maintenant et à nouveau la question de l'interprétation de l'adjectif nice attribut du sujet à droite d'un verbe copule (predicative). Considérons l'énoncé suivant :

28. This girl is nice.

52 L'adjectif appréciatif peut être prédicatif, tout comme les adjectifs descriptifs (intersectifs ou subsectifs) ; mais contrairement à l'ensemble des « adjectifs périphériques ${ }^{8}$ qui sont " attributive only » : an alleged criminal /*This criminal is alleged ; a future career/*This career is future ; presidential election/ *This election is presidential ; a southern view/ *This view is southern.

Prenons ici comme point de départ la classification des phrases copulatives de Higgins (1973) :

29. Construction prédicationnelle : Beverly is a fine baker.

30. Construction spécificationnelle : The best pie-maker is Beverly.

31. Construction à identité : Cicero is Tully.

32. Construction identificationnelle : That woman is Beverly. 
- This girl is nice - serait une construction prédicationnelle. Pour accréditer ce point de vue, on peut faire valoir que (29) - où l'adjectif fine est subsectif - a la même valeur de vérité que (33) où le prédicat cette fois est non plus un $\mathrm{SN}$ comme en (29), mais un SA comme en (28), et où le constituant frontal As a baker joue le rôle de repère restrictif :

33. As a baker, Beverly is fine. SLP

Cependant, (28) ne correspond ni à la paraphrase (34), ni à (35), mais plutôt à (36) :

34. ? ?As a person, this girl is nice.

35. ? ?As a girl, this girl is nice.

36. This girl is nice both as a girl and as a person.

Selon l'interprétation (34), le SN-sujet [This girl] en (28) n'aurait pas pour référent un membre de la classe $\mathrm{C} 1$ des [girls], mais un membre de la classe C2 des [persons]; cette possibilité s'expliquant par la notion d'/inclusion/ : C1 étant comprise dans C2 (à titre de sous-ensemble). Le SA [nice] prédicatif aurait pour cible non pas le référent propre du SNsujet [this girl], mais un référent obtenu par implication logique sur la base du principe d'inclusion. Cette paraphrase pourrait laisser entendre ceci : nice as a person, but not necessarily as a girl, cette lecture, peu évidente en soi, ne s'applique évidemment pas à l'énoncé (28). Quant à la paraphrase (35), elle laisse entendre: nice as a girl, but not necessarily as a person, ce qui est une interprétation improbable de (28). La paraphrase (36) semble plus acceptable. Cependant, elle ne peut pas représenter exactement la signification de (28), car tout repère joue un rôle restrictif, et il n'y a aucun repère de la sorte en (28).

Revenons à présent brièvement à la question du statut du SA-prédicatif [nice] - ILP ou SLP -, en comparons ce qui précède avec ceci :

37. As a coach, this person is nice. (i.e. acts nicely)

38. i.e. As a coach at least/at any rate, this person is nice.

i.e. This person has a nice way of coaching.

Le constituant repère As a coach signifie When coaching, ce qui dénote une action/activité, ce qui suggère que la prédication [(is) nice] est de type SLP ; confirmant ainsi la conclusion à laquelle nous avons abouti dans la partie 1. Ajoutons que selon le contexte et la courbe intonatoire, la proposition « this person is nice » en (37) sera ou non contrefactuelle, alors que la proposition this girl is nice ne peut l'être ni en (36), ni en (28), ce qui conforte l'idée que (36) serait une paraphrase à peu près acceptable.

\section{Prédicat d'individu (ILP) et temps verbal}

Comparons à présent :

39. ?The person I was talking to just then WAS $\left[_{S N}\right.$ a nice person].

40. The person I was talking to just then WAS ${ }_{S A}$ nice]. SLP

L'adjectif nice dans le SN-prédicatif [a nice person] de (39) est épithète prénominale : la propriété /nice/ est donc incorporée dans la représentation de l'objet dénoté par ce SN sous le signe de la «prénominalité » (comme propriété positionnelle); le SN-prédicatif désigne ainsi « un type d'individu ». Nous avons vu dans la partie 1 que le SN-prédicatif, contrairement au SA-prédicatif, serait un ILP. Soulignons à présent la compatibilité conceptuelle entre la "prénominalité » (avec son effet de préconstruction) et l'hypothèse d'un statut prédicatif de type ILP pour le SN-prédicatif. 
61 Dans les deux phrases, (39) et (40), le référent du SN-sujet [The person I was talking to] est une personne impliquée dans un procès à caractère dynamique, localisé dans le passé de l'énonciation. La prédication en (40) serait de type SLP (comme nous l'avons soutenu jusqu'ici), car ce qui fait l'objet du jugement appréciatif, c'est le comportement de la personne à un moment du passé. (40) est plus naturel que (39). Pourquoi ?

Il y aurait en (39) une certaine incompatibilité entre la composante événementielle du SN-sujet et la prédication d'individu (ILP). Il en va tout autrement en (40) ou en (41) où il n'y a aucune incompatibilité entre le SA [nice] de type SLP et la composante événementielle du SN-sujet :

41. The man/woman I was talking to WAS $\left[_{\mathrm{SA}}\right.$ nice]. (i.e. behaved nicely)

Comparons à présent (39) repris en (42) avec (43) :

42. ?The person I was talking to just then WAS [ $_{S \mathrm{~N}}$ a nice person].

43. The person I was talking to IS $\left[_{S N}\right.$ a nice person]. ILP

La différence d'acceptabilité semble conforter l'idée que le SN-prédicatif [a nice $\mathrm{N}$ ] serait de type ILP essentiellement. Si (43) est plus naturel que (42), cela tient manifestement au fait que le temps verbal est ici non plus le prétérit (was), mais le présent (is). Ce qui fait l'objet de la prédication en (43), ce n'est pas la personne telle qu'elle se serait comportée dans le temps de l'événement dénoté par la relative, mais la personne «en ellemême » dans ce qu'elle a de permanent (si l'on peut dire). La recevabilité de (43) avec une prédication ILP s'explique si l'on part du principe que la relative événementielle cette fois sert uniquement à identifier la personne thème du discours, et n'entre pas dans la portée du SN-prédicatif anaphorique [a nice person]. Ainsi, la cible de la prédication en (43) ne serait pas « la personne à qui je parlais telle qu'elle était il y a un instant ", mais plutôt « la personne à qui je parlais telle qu'elle est toujours » (selon l'énonciateur); ce qui est signifié conjointement par le temps verbal (présent) et la prédication d'individu (ILP). Une implication supplémentaire de cette analyse, basée sur le postulat que le SNprédicatif [a nice person] est bien un ILP, est que la connaissance de la nature de la prédication (ILP) suffit pour inférer que le locuteur de (43) connaît la personne depuis un certain temps.

L'acceptabilité incertaine de (42) quant à elle s'expliquerait alors par le fait que la marque du temps passé sur le verbe be (was) signifie que le jugement appréciatif porte spécifiquement sur la personne telle qu'elle se comportait pendant le temps de l'événement décrit dans le SN-sujet. Dans ces conditions, le prédicat devrait être de type SLP, donc préférablement le SA [nice], et non le SN-ILP [a nice person].

\section{Lecture intersective/subsective de la construction "SN is [an AN] »}

La construction prédicationnelle " $\mathrm{SN}$ is $[a n$ AN ]» reçoit une interprétation intersective s'il est vrai que : 1 . SN is $\mathrm{A}$, et $2 . \mathrm{SN}$ is $\mathrm{N}$ :

44. Mike is a nice football player. (nice as a person)

Nous comprenons que : 1. Mike is nice, 2. Mike is a football player, mais pas que : \#Mike plays football nicely. (44) est cependant une exception, car avec la construction « SN is [a nice N] ", la lecture intersective n'est pas toujours disponible :

45. ? ?Jane is a nice driver.

a. \#Jane is nice. 
b. Jane is a driver.

c. \# Jane drives nicely.

68 On observe également que la lecture subsective (45c) n'est pas non plus disponible. Pour signifier (45c), il faut remplacer nice par l'adjectif good ${ }^{9}$ et aboutir ainsi à une construction non intersective :

46. Jane is a good driver.

\# Jane is good.

Jane is a driver.

Jane drives well (in a good way)

69 Le SN [a good book] signifie quelque chose comme a book that makes good reading/that is worth reading/that one enjoys reading. Le SN [a good person] signifie a person who does good things/who acts in a good way. Si A= good, l'interprétation du couple AN implique nécessairement un procès, donc une structure verbale (Vendler, 1963).

70 Avec l'adjectif beautiful, hors contexte, l'interprétation d'une phrase comme (47) serait ambiguë :

47. Olga is a beautiful dancer

beautiful as a woman lecture intersective

beautiful as a dancer lecture non intersective

71 La construction prédicationnelle « SN is [a nice N] » reçoit le plus souvent une lecture subsective (non intersective) où l'incidence de l'adjectif nice et sa portée coïncident, contrairement à ce qu'il se passe avec l'adjectif beautiful qui est compatible avec la lecture intersective de la phrase (A portant sur le SN-sujet). Voici quelques exemple de constructions subsectives :

48. Jane is a nice coach.

49. Sam is a nice collaborator.

50. They are nice neighbours.

Nous comprenons immédiatement que a nice coach signifie someone who coaches nicely, a nice collaborator, someone who collaborates nicely, et nice neighbours, people who act nicely as neighbours. Cependant, avec nice, souvent la lecture subsective ne parvient pas à occulter la lecture intersective. Ainsi, en (48) par exemple, nous comprenons la prédication adjectivale comme ayant pour cible à la fois le nom coach et le SN-sujet Jane (as a person). De tels exemples montrent que dans la construction prédicationnelle « SN is [a nice $\mathrm{N}]$ ", nice peut avoir une portée double.

73 À titre de comparaison, signalons que cette double portée n'est pas réalisable avec l'adjectif good:

51. They are good neighbours.

good as neighbours. lecture subsective (uniquement)

\# good as people. lecture intersective (non disponible)

\section{Nice et le concept de « bon objet»}

74 Examinons à présent deux exemples (dont un déjà vu) de SN comprenant plus d'un adjectif épithète :

52. BYU-BNC $200 \mathrm{HT} 3 \mathrm{~W}$-advert : ... heating and water heating will turn itself on. So you come home to a nice warm, brightly lit home with plenty of piping hot water to soak-away the dust. 
53. COCA : 418 BLY : Starting from this distinction it is easy, and initially satisfying, to present a nice clear argument, supported by well-chosen anecdotes, proving how wide and how unacceptable ... notion nominale se fait de façon positive, et dénote ainsi ce que l'on pourrait appeler (par commodité pour la suite) « un bon objet». Or l'adjectif nice est souvent suivi d'un ou de plusieurs autres adjectifs épithètes, comme en (52) et (53), lesquels ont pour fonction de conditionner la représentation en passant sous silence toutes les autres virtualités entrant par ailleurs et ordinairement dans la représentation prototypique du «bon objet ». Ainsi par exemple, le SN [a nice long walk] signifie une promenade agréable (nice) car «longue». Or on sait bien que dans la notion complexe /nice walk/, les propriétés positives entrant dans la représentation ne se limitent pas à la «longue durée » de la promenade.

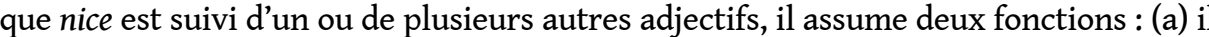
signale que le SN en cours de construction va dénoter un «bon objet»; (b) il signale de façon synthétique que l'objet est bon en particulier en vertu des propriétés dénotées par les autres adjectifs. La fonction (a) se comprend dans le temps et le sens de la lecture, orientée de gauche à droite; tandis que la fonction (b) se comprend dans la perspective inverse, de droite à gauche en partant de N. La fonction (b) implique donc une lecture inversée.

81 Il arrive que la fonction (a) puisse être privilégiée, en plaçant une virgule à droite de A1 (nice) :

54. COCA : 21492015 SPOK NPR : well, nine people but seven kids in a very small house, [a very nice, neat, clean, little house], but a small house. 
la différence des deux exemples précédents, A1 (nice: adjectif appréciatif) et A2 (plus descriptif) sont séparés par une virgule. En règle générale, la virgule dans les séquences adjectivales signale une incidence disjointe. Ainsi, les SA very nice, neat, clean et little sont à cet égard mis sur le même plan, même si les trois adjectifs qui suivent nice appartiennent à une autre classe d'adjectifs, étant plus "descriptifs ». Cependant, là comme ailleurs, c'est bien l'adjectif appréciatif qui occupe la position A1. Il semblerait que la présence de la virgule après nice permet de privilégier la fonction (a). La virgule implique une pause et permet de mettre l'adjectif nice en évidence en le dissociant (en le désolidarisant momentanément) des adjectifs descriptifs qui le suivent. Pendant la durée de cette pause, la fonction (a) est pleinement perçue.

\section{Conclusion}

Nice appartient à la classe des adjectif appréciatifs; il signifie que l'objet en question produit (ou est susceptible de produire) un effet positif, voire agréable. Nice a pour fonction immédiate de convoquer l'idée abstraite d'un objet idéal. Si aucun autre adjectif ne sépare nice de la tête nominale $\mathrm{N}$ (support de l'incidence), et si N n'est pas postmodifié, le couple AN à lui seul convoque l'image d'une occurrence idéale de la notion (ce que l'on a nommé ici un «bon objet»). Cette image incorpore à la représentation de l'objet des caractéristiques accidentelles positives, en principe en nombre pluriel, mais laissées dans l'implicite. On en conclut que nice assume une fonction synthétique, même si le SN ne comprend aucun autre adjectif.

(en anglais) lui permet de signifier que le référent du couple AN est un type d'objet préconstruit, si bien que l'énonciateur ne fait que l'actualiser dans la désignation d'un objet particulier ou générique. L'adjectif nice étant gradable, la possibilité d'une modalisation (quite a nice place) montre que l'attribution de la qualité /nice/ n'est pas soumise à un schéma conceptuel strictement dichotomique du genre : /nice/ versus /not nice/.

Dans la partie 1, trois tests classiques de prédication - permettant de distinguer le prédicat épisodique (stage level predicate) du prédicat d'individu (individual level predicate) - ont indiqué que le SA [nice] en position d'attribut du sujet (predicative) serait un prédicat épisodique (SLP), et qu'à l'inverse, le SN-prédicatif [a nice $\mathrm{N}$ ] serait un prédicat d'individu (ILP).

Dans la construction prédicationnelle « SN is [a nice $\mathrm{N}$ ] », le SN-prédicatif [a nice N] a aussi été examiné en variant le paramètre du temps verbal sur le verbe be (was $\rightarrow$ is). On a montré que, paradoxalement, ce prédicat de type ILP n'est pas incompatible avec un SNsujet dont la tête nominale $\mathrm{N}$ est complétée par une relative à contenu événementiel : The person I was talking to IS a nice person. La conclusion ici était que le SN-prédicatif [a nice person] porterait non sur le référent propre du SN-sujet (la personne en fonction d'un comportement à un moment donné), mais sur la personne "en elle-même » une fois identifiée par la relative; l'implication étant qu'une prédication ILP peut occulter la composante événementielle d'un SN-sujet dès lors que celle-ci a fini de jouer le rôle d'identification qui lui est assigné.

En se basant sur les concepts d'intersectivité/subsectivité, il a aussi été montré que la construction prédicationnelle « $\mathrm{SN}$ is [a nice $\mathrm{N}]$ » peut recevoir trois lectures différentes : une interprétation exclusivement intersective (impliquant pour nice une dissociation 
entre portée et incidence) ; plus souvent, une interprétation subsective (nice portant sur la tête nominale du SN-prédicatif); et parfois les deux interprétations, ce qui implique pour nice la possibilité d'une portée double.

\section{BIBLIOGRAPHIE}

Arigne, V., 2015. La modalité appréciative : vers une sémantique de good, Anglophonia [Online], N ${ }^{\circ} 19$ | 2015. http://anglophonia.revues.org/509;DOI: 0.4000/anglophonia.509

Bolinger, D., 1967. Adjectives in English: Attribution And Predication, Lingua 18, Amsterdam, North-Holland Publishing Co.. 1-34.

Carlson, G., 1980. Reference to Kinds in English. New York: Garland. (1977 PhD dissertation, university of Massachusetts).

Greenbaum S. \& R. Quirk, 1990. A Student's Grammar of the English Language, Longman.

Hadermann P., Pierrard M., Van Raemdonck D., 2007. La scalarité : autant de moyens d'expression, autant d'effets de sens, Travaux de linguistique 2007/1, $N^{\circ} 54$. 7-15. http:// biblio.ugent.be/publication/445725/file/6814310.pdf

Higgins, F., 1973. The Pseudo-Cleft Construction in English, Thesis, MIT.

Jager G., 1999. Stage levels, states, and the semantics of the copula, Zentrum fur Allgemeine Sprachwissenschaft, ZAS Papers in Linguistics 14. 65-94. http://www.sfs.uni-tuebingen.de/ gjaeger/publications/zwpl.pdf

Kertz, L., 2006. Evaluative Adjectives: An Adjunct Control Analysis, Proceedings of the $25^{\text {th }}$ West Coast Conference on Formal Linguistics, ed. Donald Baumer, David Montero, and Michael Scanlon. 229-235. www.lingref.com/cpp/wccfl/25/paper1453.pdf

Thomas, I., L'étiquetage sémantique des adjectifs qualificatifs en vue de leur désambiguïsation dans les groupes nominaux simples. www.atala.org/doc/JE_050312/Lexsynt-Thomas.pdf

Vendler, Z., 1963. The Grammar of Goodness, The Philosophical Review, vol. 72, N 4. 446-465.

Yagi, K. 2006. Patterning of English Adjectives - For the Improvement of Learners' Dictionaries, Asian Bilingualism and the Dictionary. Proceedings of The Second International Congress, Konsei University, Seoul, Korea: 112-118.

\section{NOTES}

1. Cf. Carlson (1980). Pour un aperçu rapide : cf. Jager (1999).

2. Voici une liste d'adjectifs appréciatifs proposée par Barker (2002) : brave, careless, clever, (in)considerate, courageous, cowardly, crazy, cruel, dumb, evil, foolish, impudent, (un)kind, (un)lucky, mean, naughty, nice, (im)polite, (im)prudent, right, silly, smart, stupid, wicked, (un)wise, wrong.

3. Kertz (2006) s'interroge sur la nature exacte de la prédication lorsque le prédicat est un adjectif appréciatif (evaluative) suivi de la structure infinitive [to V]. 
4. Les «adjectifs d'attitude» sont parfois désignés «adjectifs dispositionnels " (disposition adjectives) : eager, keen, willing, unwilling .... Ces adjectifs requièrent un complément, lequel prend la forme d'une structure infinitive qui dénote un procès localisé ou localisable dans le temps. La disposition mentale exprimée par l'adjectif est en relation exclusive avec ce procès, ce qui explique que la prédication est de type SLP.

5. Hadermann \& al. 2007 : «En sémantique le terme «scalaire » a été associé non seulement à des prédicats mais aussi à des déterminants, des adjectifs, des noms ainsi qu'à des expressions adverbiales gradables. La constante qui caractériserait tous ces domaines d'application serait qu'un terme scalaire (...) impliquerait une référence à des niveaux partiellement ordonnés sur une échelle selon une certaine propriété qui permet la gradation [3]».

Note $3:$ « D'après le T.L.F., la gradation désigne la progression d'un état vers un autre par paliers insensibles et généralement ascendants ».

6. Une position syntaxique est munie d'une propriété positionnelle si tout constituant occupant cette position hérite d'une propriété toujours associée à cette position.

7. I. Thomas (2015) propose une « catégorisation générale des adjectifs qualificatifs en cinq types de Propriétés, définies en fonction de la nature de l'objet qu'il catégorise : a) Propriétés des objets matériels, b) Propriétés des objets vivants, c) Propriétés des humains, d) Propriétés des abstraits, e) les adjectifs non-spécifiques (ANS), c'est-à-dire ceux qui peuvent décrire les propriétés de n'importe quel objet, indépendamment de leur statut catégoriel. »

8. Dans un SN comprenant plusieurs adjectifs, les adjectifs non inhérents jouant un rôle de type localisation sont placés juste après le déterminant; et les adjectifs ou les noms à fonction classifiante sont placés juste devant $\mathrm{N}$ : [HER (det) last very pretty dark blue evening DRESS (N)].

9. Sur l'adjectif good, voir notamment Vendler (1963) et Arigne (2015).

\section{RÉSUMÉS}

Cette étude porte sur l'adjectif nice dans une analyse synchronique ; adjectif dont la signification (en discours) varie selon le contexte à tel point qu'il occupe une place exceptionnelle dans la classe des «adjectifs appréciatifs». Cet article focalise sur des questions d'interprétation, en comparant plusieurs emplois et structures. L'analyse est basée principalement sur deux couples de concepts : l'opposition entre " prédicat d'individu » et " prédicat épisodique » (individual/stage level predicate: ILP/SLP), et l'opposition entre interprétation "intersective » du prédicat et interprétation «subsective" (non intersective); avec pour objectif plus général de montrer l'utilité de ces outils conceptuels dans l'étude notamment des « adjectifs appréciatifs».

S'agissant spécifiquement de l'adjectif nice, on fait valoir ici que les SA-prédicatifs [nice] et [nice [with SN]] seraient des prédicats épisodiques (SLP), alors que le SA-prédicatif [nice [of SN]] ainsi que le SN-prédicatif [a nice $N$ ] seraient des prédicats d'individu (ILP). On montre ensuite que, paradoxalement, dans la construction prédicationnelle « SN is [a nice N] », le SN-prédicatif [a nice $N]$ de type ILP n'est pas toujours incompatible avec un SN-sujet ayant une composante événementielle. On montre enfin que la construction « $\mathrm{SN}$ is [a nice $\mathrm{N}]$ 》 peut recevoir trois lectures différentes selon le contexte: une interprétation intersective par laquelle nice porte uniquement sur le $\mathrm{SN}$-sujet (impliquant une dissociation entre portée et incidence); plus souvent, une interprétation subsective (A portant uniquement sur la tête nominale cible de l'incidence); une lecture qui superpose les deux interprétations, ce qui implique pour nice la possibilité d'une portée double. 
This article is an analysis of the various uses of the qualifying adjective nice: as an attributive adjective standing alone in front of the head noun, or completed by a PP : [with NP] or [of NP] ; as a predicative adjective, and it is then compared with the predicative NP [a nice N]. It is here argued that the predicative APs [nice] and [nice [with NP]] are both stage level predicates (SLP), while the predicative AP [nice [of NP]] and the predicative NP [a nice N] are individual level predicates (ILP). Paradoxically, the latter ILP predicate - i.e. [a nice N] as subject complement - is then shown to be sometimes compatible with an NP-subject whose head noun is postmodified by an eventive relative clause. It is also claimed that the construction «NP is [a nice N] » can have three different readings: an intersective one in which nice qualifies the NP-subject; more often a subsective reading in which nice qualifies the head noun of the predicative NP; or a double reading, when the subsective reading does not exclude an additional intersective one.

INDEX

Mots-clés : adjectif appréciatif/descriptif, épithète/attribut, prédicat d'individu/épisodique, interprétation intersective/subsective.

Keywords : evaluative/descriptive adjectives, attributive/predicative adjectives, individual level/stage level predication, intersective/subsective reading.

\section{AUTEUR}

\section{CHRISTOPHER DESURMONT}

Université Charles-de-Gaulle, Lille 3

Laboratoire STL (UMR 8163)

c_desurmont@hotmail.com 